\title{
Numerical Simulation of Hypersonic Flow over a Blunt Wedge in Response to Impulse Wave
}

\author{
Xiaojun Tang ${ }^{\text {a, }}{ }^{*}$, Fenglong Yang ${ }^{a}$, Tianli Hui ${ }^{a}$, Wentao $\mathrm{Yu}^{\mathrm{a}}$ and Pengbo Yin ${ }^{\mathrm{a}}$ \\ Beijing Spacecrafts, China Academy of Space Technology, Beijing 100094, China \\ *axiaojuntang87@sina.com, bfenglongy@sina.com, chuitianl@sina.com, dwentaoy@aliyun.com, \\ epengboyin2013@163.com
}

Keywords: Direct Numerical Simulation; impulse wave; hypersonic flow; boundary layer.

\begin{abstract}
The hypersonic flow over a blunt wedge under freestream impulse wave is analyzed by direct numerical simulation and the nonlinear evolution of disturbance wave modes in the boundary layers is discussed. Results show that, under impulse wave, the thermodynamic mechanism and shear flow structure in the boundary layer is sharply changed, and the effect of the reflection wave between bow shock and wall on shear flow structure is larger than that of freestream impulse wave. Both the disturbance waves with frequency less than fundamental mode and the harmonic modes in hypersonic boundary layer are induced under impulse wave. Flow recompression plays a significant role in the evolution of disturbance waves in the boundary layer. Low frequency disturbances are the dominant mode in the nose boundary layer. With the evolution of disturbance waves along streamwise, the boundary layer will be dominated by the second harmonic mode $(x>2.3)$, and all disturbance modes decrease along streamwise except the second order harmonic mode $(x>4.5)$.
\end{abstract}

\section{Introduction}

Disturbances widely exist in flying environment, and flying vehicle designs are often limited by the effect of disturbance on aerothermodynamics properties as well as aircraft structural strength [1] due to the fact that an unsteady flow generates much more uncertainties and much higher drag as well as wall heat flux than a steady one. Especially, strong disturbance plays an extremely important role in determining the lift, drag and heat-load on surface. Because of disturbances in flowfield, the accurate prediction of aerodynamic force, drag and thermal state present a serious challenge, which is the development bottleneck of aircraft. Therefore, it is essential to understand the interaction mechanism between the disturbance wave and flow, and the influence of disturbance on flowflied. Some numerical simulations have been performed on the effects of disturbance in the past few decades [2-4], but most of these are based on turbulence models. The unsteady flowfield, especially the flow with strong disturbance wave maintains different stability characteristics under different flow conditions, which cannot be accurately modeled by particular turbulence model. Also many researches have been conducted on hypersonic flow stability based on disturbance [5-13], such as freestream disturbance wave [5-9] and wall blowing-suction [10-13]. As shown in the previous, it has been confirmed that the disturbance in flowfield affects the properties of aerothermodynamics, and most of these efforts focused on the effects of continuous small disturbance on aerothermodynamics properties of hypersonic flowfield and boundary layer stability characteristic using direct numerical simulation (DNS) and linear stability theory (LST). However, few researches were conducted on the effects of strong disturbance with limited length of time on aerothermodynamics properties of hypersonic flowfield and boundary layer stability characteristic. It is obviously that the aerothermodynamics properties and boundary layer stability characteristic under continuous disturbance is also rather different from that under the disturbance with limited length of time. Thus, it can be concluded that the aerothermodynamics properties of hypersonic aircraft is affected by the strong disturbance in hypersonic flow, and the influence mechanism of impulse disturbance (the disturbance with limited length of time) on hypersonic flowflied is still not understood. To make excellent design of flying 
vehicles, and to understand the response of hypersonic flow to impulse wave, it is essential to focus on the interaction between impulse wave and shock wave as well as boundary layer.

In this paper, direct numerical simulation of hypersonic unsteady flow over a blunt wedge in response to freestream impulse wave is conducted. The interaction between the impulse wave and hypersonic flowfield is analyzed. The evolution of disturbance wave modes in boundary layers, boundary layer stability, and the effect of impulse wave on aerothermodynamics properties are investigated.

\section{Numerical methods}

Direct numerical simulation of hypersonic flow over a blunt wedge in response to impulse wave is conducted in this paper. For the convenience of calculation, the two dimensional compressible Navier-Stokes equations at Cartesian coordinates are transformed into curvilinear coordinates. The governing equations for compressible flow can be expressed as follows:

$$
\frac{\partial\left(\mathbf{J}^{-1} \mathbf{U}\right)}{\partial t}+\frac{\partial \mathbf{E}^{*}}{\partial \psi}+\frac{\partial \mathbf{F}^{*}}{\partial \phi}+\frac{\partial \mathbf{E}_{v}^{*}}{\partial \psi}+\frac{\partial \mathbf{F}_{v}^{*}}{\partial \phi}=0
$$

Where $\mathrm{J}$ is Jacobin transformation matrix; $\mathbf{E}^{*}$ and $\mathbf{F}^{*}$ are no-viscid terms corresponding to two axial directions at general curvilinear coordinates; $\mathbf{E}_{v}^{*}$ and $\mathbf{F}_{v}^{*}$ are viscid terms corresponding to two axial directions at general curvilinear coordinates. While

$$
\begin{cases}\mathbf{E}^{*}=\frac{\mathbf{E} \psi_{x}+\mathbf{F} \psi_{y}}{\mathbf{J}} & \mathbf{F}^{*}=\frac{\mathbf{E} \phi_{x}+F \phi_{y}}{\mathbf{J}} \\ \mathbf{E}_{v}^{*}=\frac{\mathbf{E}_{v} \psi_{x}+\mathbf{F}_{v} \psi_{y}}{\mathbf{J}} & \mathbf{F}_{v}^{*}=\frac{\mathbf{E}_{v} \phi_{x}+\mathbf{F}_{v} \phi_{y}}{\mathbf{J}}\end{cases}
$$

Where $\mathbf{U}$ is the state vector; $\mathbf{F}$ and $\mathbf{G}$ are no-viscid terms. $\mathbf{F}_{\mathrm{v}}$, and $\mathbf{G}_{\mathrm{v}}$ are viscid terms. Compressible Navier-Stokes equations are discretized spatially and temporally by high-order finite difference methods for direct numerical simulations of hypersonic flowflied. Stager-Wariming splitting method [14] is introduced for the splitting of convection terms. Fifth order upwind WENO scheme [15] is employed for the discretization of convective terms; Sixth order central finite-difference scheme [16] is used for the discretization of viscous flux terms; third order Runge-Kutta TVD type method [17] for time advance. It should be noted that the numerical simulations in our previous paper [18] have been well validated by Stetson's experiment [19] and the numerical results of A. Prakash et al [20] and Zhang et al [21]. It is reasonable to believe that the numerical code is reliable.

\section{Flow conditions and model}

To investigate the effects of freestream impulse wave on hypersonic boundary layer stability characteristics and aerothermodynamics of hypersonic flowfield, DNS are performed for hypersonic flows over a blunt wedge. No-slip and adiabatic wall conditions are enforced at the wall surface, and symmetry condition, freestream condition and extrapolation boundary condition are employed at the $\mathrm{x}$-axis, upstream boundary and downstream boundary, respectively. The solutions of hypersonic unsteady flowfield are conducted by $300 \times 120$ grids. The mesh grid in this paper is the same with that in our previous paper [18] have been well validated. The Reynolds number, $\operatorname{Re}=\rho_{\infty} r u_{\infty} \mu_{\infty}$, based on freestream parameters and the wedge's nose radius, is equal to 6000 , where $\rho_{\infty}, r, u_{\infty}$ and $\mu_{\infty}$ are the density, the wedge's nose radius, freestream velocity and freestream viscosity coefficient, respectively. Subscripts ' $\infty$ ' denotes freestream condition. The parameters used in the paper are dimensionless, and the details are expressed as follows: $u / u_{\infty}, v_{\infty} / u_{\infty}, l / r, \mathrm{P} / \rho_{\infty} u_{\infty} u_{\infty}$, and $t /\left(r / u_{\infty}\right)$, where $t, u, v, l, \mathrm{P}$ and T are the time, velocity along $x$-axis, velocity along $y$-axis, length, pressure and temperature in flow field, respectively. The freestream Mach number $M_{\infty}=6$, and the freestream temperature $T_{\infty}=69 \mathrm{~K}$. The wedge's nose radius $r=1 \mathrm{~mm}$; the angle of attack $\alpha=0^{\circ}$; the half wedge angle $\theta=8^{\circ}$. The disturbance 
fields are represented by instantaneous flow variables minus the local steady base flow variables at the same location, which can be expressed as follow [21].

To facilitate analyzing the evolution of the impulse wave in the hypersonic boundary layer, the impulse wave is considered as the slow acoustic wave with a half sinusoidal form. To do direct numerical simulation of hypersonic unsteady flow, the impulse wave is introduced at the upstream boundary at $t=0.0$. The form of the impulse wave can be expressed as follow:

$$
\begin{cases}\mathbf{q}^{\prime}=\mathbf{q} \mathrm{e}^{\mathrm{i}\left(k x-\frac{F \cdot \operatorname{Re}}{10^{6}} t+\frac{\pi}{2}\right)} t \leq 2.0 \\
\mathbf{q}^{\prime}=\left[\begin{array}{llll}
0 & 0 & 0 & 0
\end{array}\right]^{T} \quad t>2.0\end{cases}
$$

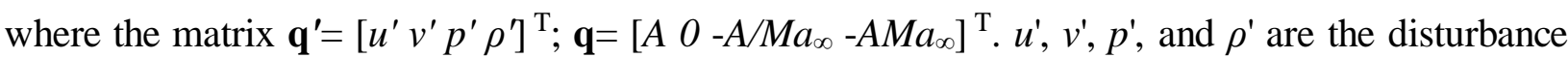
values of velocity along $\mathrm{x}$-axis, velocity along $\mathrm{y}$-axis, pressure and density, respectively; amplitude $A=0.08$, and wavenumber $k=3.1446 \times 10-4$. Generalized frequency $F=50 \pi$, which corresponds to dimensionless frequency $f=0.25$.

\section{Results and discussion}

In the present study, the interaction process between freestream impulse wave and hypersonic flowfield are simulated for investigating its effect on aerothermodynamics properties. Temperature disturbance contours varying with time are shown in Figs.1. Fig.1(a), (b), (c) and (d) correspond to $t=2.5,4.5,6.5$ and 8.5, respectively. It can be seen that, after the interaction between impulse wave and bow shock wave, the temperature disturbances is different from freestream temperature disturbances sharply. There is significant temperature disturbance difference between outside and inside the boundary layer, which shows more complex interactions inside the boundary layer. Obviously, under freestream impulse wave, the boundary layer thermal conduct mechanisms are different from the ones before. The freestream wave will change the stability characteristic of boundary layer since thermal conduct mechanism plays an important role on boundary layer stability [22, 23].

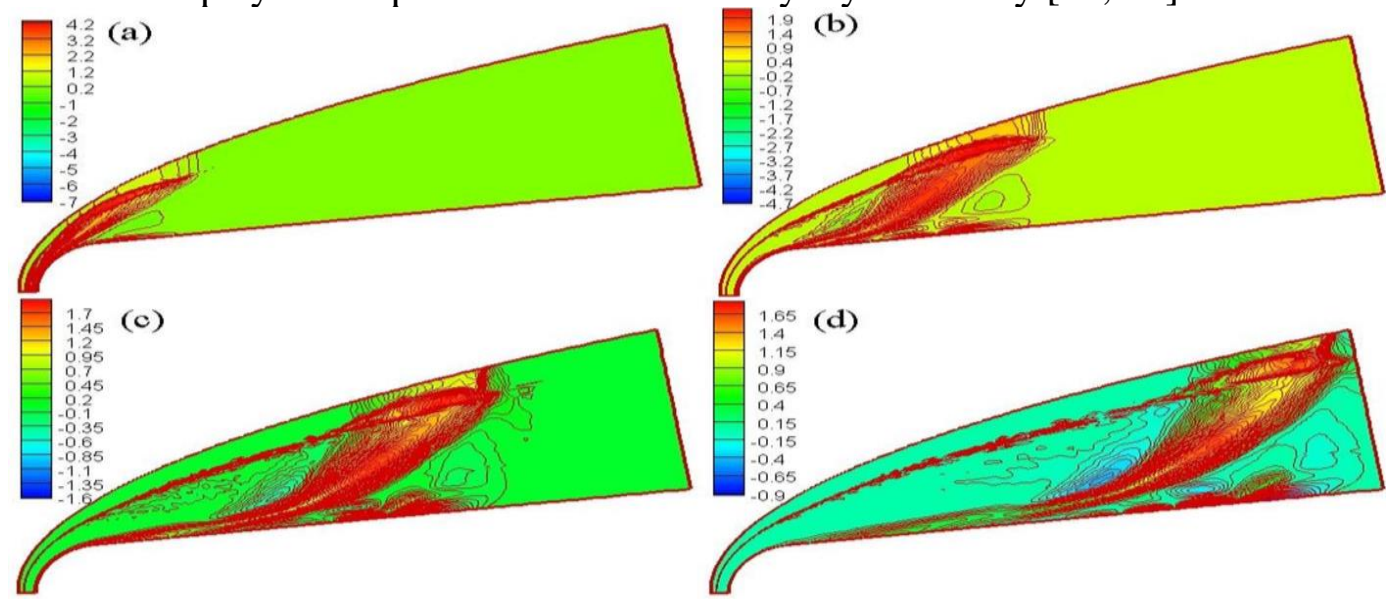

Figure 1. Contour of temperature at different times

Contours of velocity along y-axis at $\mathrm{t}=0.0,3.8,6.2$ and 7.4 are shown in Fig.2(a), (b), (c) and (d), respectively. It is found clearly that the velocity along y-axis (stream wise) of flowfield changes with time during the interaction process. The velocity along y-axis in boundary layer changes sharply, and the negative velocity can be found, which indicates there exists eddy near the wall surface region under freestream impulse wave. It also can be seen that the interactions between freestream wave and bow shock are very strong. The bow shock is bent obviously outwards, and the location and shape of bow shock wave changes sharply during the interaction process. Meanwhile, it is also observed that the velocity along $\mathrm{y}$-axis rise significantly in the convex area caused by the interaction between the freestream wave and the bow shock, as the mark N shown in Fig.2. 


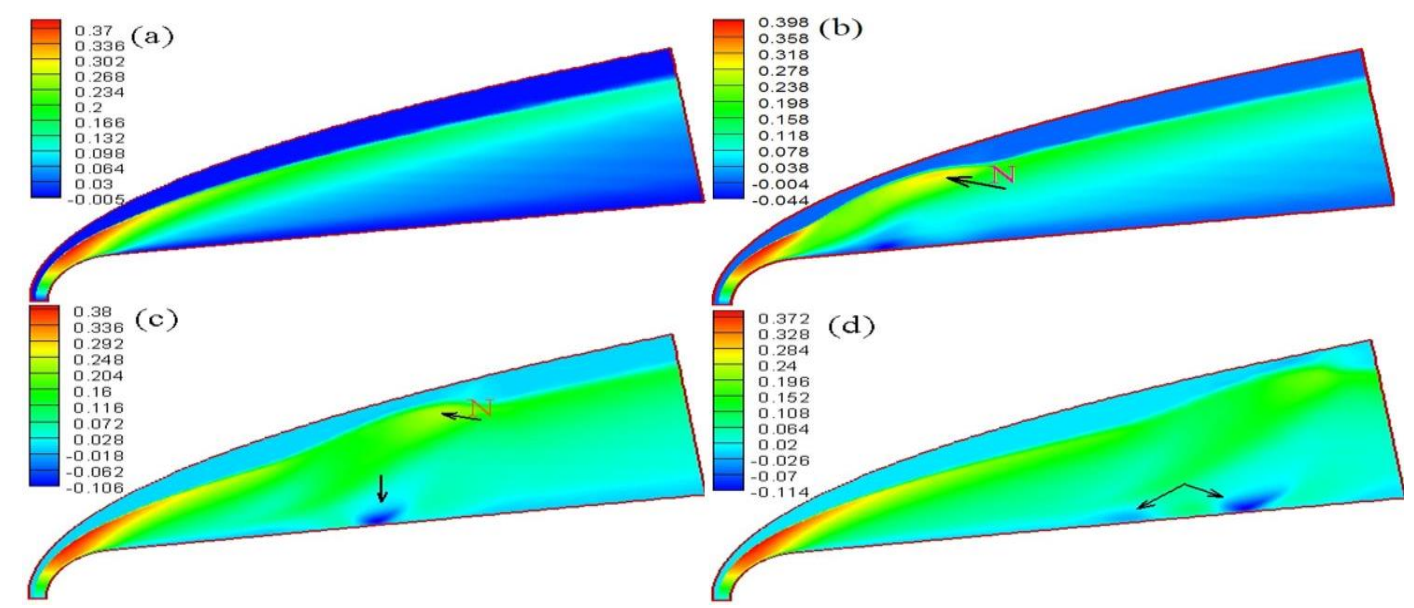

Figure 2. Contour of velocity along $y$-axis $v(x, y, t)$ at different times

It should be mentioned that, to investigate the effects of strong disturbance wave on aerothermod ynamics parameters, four grid lines normal to the surface are chosen $(x=-0.50164,0.00237,0.98893$, 2.02224). Meanwhile, in order to vividly show variation of the aerothermodynamics parameters on the four lines, 10 time points are chosen. Variation of the velocity (along $y$-axis) on the grid lines normal to the wall surface are shown in Fig.3. It can be seen that the velocity along y-axis changed with the time during the perturbation process, which is an oscillation process. The variation of velocity along y-axis on different grid lines is similar to each other. It firstly undergoes a rising and decline, then becomes large slightly, and then undergoes a further decline along the lines, which is an oscillatory process that the amplitude of oscillation becomes gradually smaller. It should be mentioned that the process is the evolution process of disturbance wave in hypersonic flowfield. It also can be clearly observed that the variation of shock wave location in the oscillation process is similar to a damped motion, as the mark A1 shown in Fig.3. Meanwhile, it can be seen that that, during the oscillatory process, negative velocity along $y$-axis can be found near wall surface when being subjected to freestream wave, the reason for which is believed to be that there is eddy.
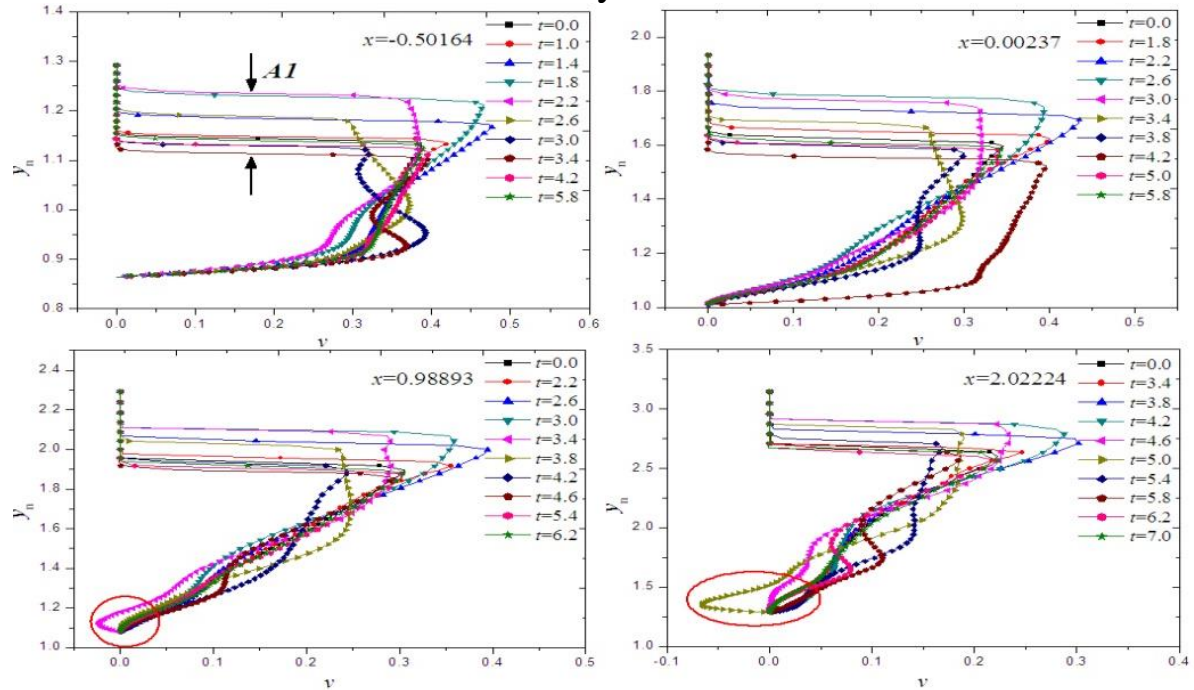

Figure 3. Variation of velocity along y-axis at four grid stations

Fig. 4 shows the variation of temperature on the grid lines normal to the wall surface at two grid stations ( $x=-50164,0.00237)$. From Fig. 3 and 4, the trend of temperature is similar to that of velocity along y-axis, which is an oscillation process. It can be found that the variation of shock location and freestream temperature disturbance, as the mark A1 and A2 shown in Fig.4. It also can be seen that wall temperature changed with the time under freestream impulse wave, as the mark A3 shown in Fig.4. It should be mentioned that there is a strong interplay between the thermal state on wall and the boundary layer state, which exerts an important impact on flow stability and boundary layer transition [24]. 

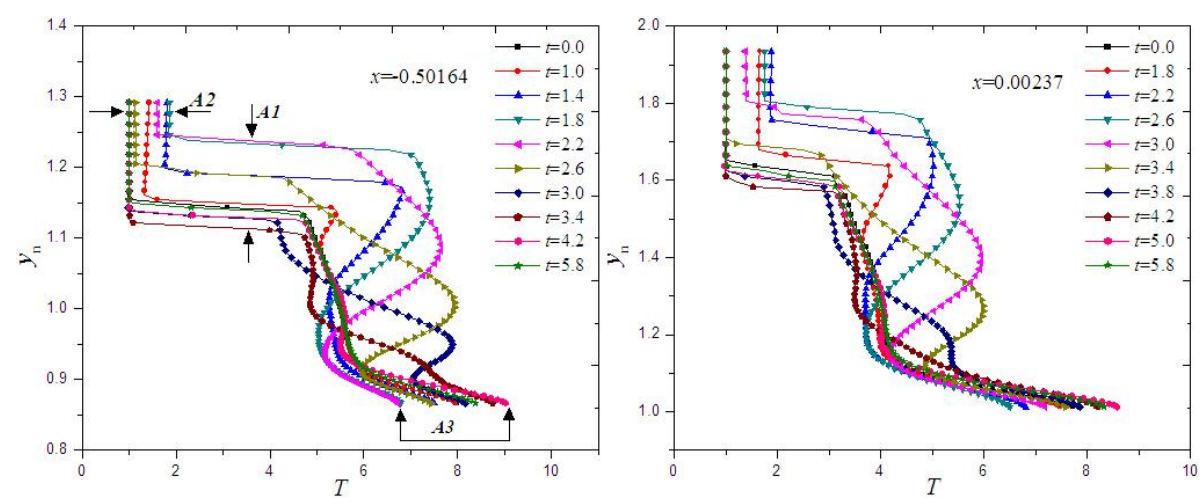

Figure 4. Variation of temperture at two grid stations

Fig. 5 shows the distributions of the friction factor $f(\mathrm{x}, \mathrm{y}, \mathrm{t})$ on wall at different times $(t=0.0,2.0,3.0$, 4.0, 5.0, 6.0, 7.0, 8.0 and 9.0). It is found that, under the action of the impulse wave (freestream disturbance wave), the friction factor $f(x, y, t)$ on wall becomes large firstly(as the mark f1 shown in Fig.5), and then becomes smaller(as the mark f1 shown in Fig.5), the shape of which is are similar to the disturbance in freestream. When the impulse wave passes through entirely, the variation of disturbance amplitude of friction factor is a damped oscillation process till the change of $f(x, y, t)$ vanishes. It is believed that the reason for the phenomenon due to that after freestream disturbance wave interacting with bow shock, a part of oscillating disturbance waves will move back and forth between bow shock wave and wall [25]. It also can be seen that the distuabnce amplitude flis smaller than $\mathrm{f} 2$, as shown in Fig.6. It can be concluded that shear flow structure is affected by freestream wave, and the effect of reflection wave on shear flow structure is larger than that of freestream wave.

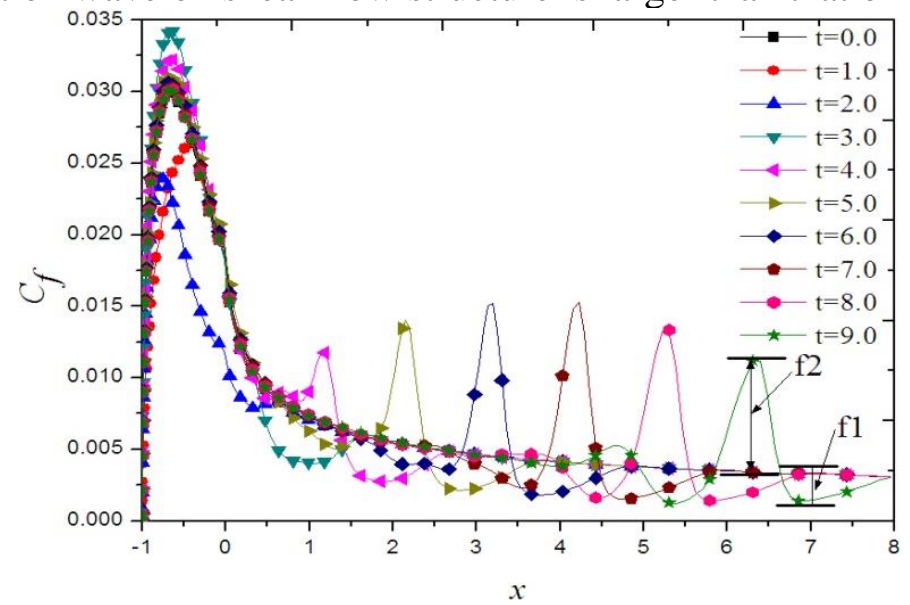

Figure 5. Comparison of friction factor profile along streamwise at different times

In order to investigate the wave's modes in the blunt wedge boundary layer and their evolution, the pressure disturbance in boundary layer (on wall surface) is decomposed from time domain signal to the frequency domain signal by Fourier transform. Fig.6 shows the evolution of pressure disturbances amplitudes for fundamental mode $\left(f_{1}=0.25\right)$ and less than fundamental mode $(f<0.25)$ in boundary layer along streamwise. It can be seen that, under the action of freestream impulse wave with $f=0.25$, there are disturbance modes with frequency less than fundamental mode in boundary layer. When $s>\pi / 2$ (in no-nose boundary layer), the amplitude of fundamental mode is larger than that of the disturbance modes with frequency less than fundamental mode; the amplitude of fundamental mode and the disturbance modes with frequency less than fundamental frequencies keep decreasing along streamwise, however the decay rates of which become small; meanwhile there are some modes with larger amplitudes. This means that the disturbance modes with frequency less than fundamental mode play an important role in determining the stability characteristic of hypersonic boundary layer over a blunt wedge under freestream impulse wave. Fig.7 shows the evolution of pressure disturbances amplitudes for harmonic frequencies $\left(f_{2-8}\right)$ in boundary layer along streamwise. From Fig.6 and Fig.7, it can be seen, 1) after the freestream wave interacting with bow shock and then entering the boundary layer, both the disturbance waves with frequency less than fundamental modes $(f<0.25)$ and harmonic 
frequency modes in boundary layer are induced. 2) In the nose boundary layer, all modes (both fundamental mode and harmonic modes) decrease rapidly along streamwise. Obviously, this is the result of normal shock becoming oblique shock. In the no-nose boundary layer, all harmonic modes have a rise when $0<s<4$, however, fundamental mode $\left(f_{1}=0.25\right)$ and the disturbance with frequency less than fundamental mode $(f<0.25)$ in boundary layer along streamwise still keep decreasing although the decay rates becoming small. It should be mentioned that, due to the surface shape, the hypersonic flow is recompressed in the junction region between the nose and the straight wedge [25]. We prefer to maintain that the rise of harmonic modes and the decay rates of low frequency $(f \leq 2.5)$ becoming small caused by the flow recompression, or at least the recompression plays a significant role in the evolution of disturbance waves in boundary layer. 3) In nose boundary layer, the amplitudes for fundamental mode $\left(f_{1}=0.25\right)$ and the disturbance mode with frequency less than fundamental mode $(f<0.25)$ are significantly larger than that of harmonic modes; whereas; when $x>2.3$, the amplitude of the second order harmonic mode is larger than that of fundamental mode, which indicates the dominant mode in boundary layer is transferred from the fundamental mode to the second order harmonic mode. However, there are some disturbance modes with lager amplitudes in boundary layer, such as the modes with $f=0.20, f=0.15$, the third order harmonic mode, the forth order harmonic mode and the fifth order harmonic mode; these disturbance modes are still the main disturbance modes in boundary layer. 4) As disturbances evolution from upstream to downstream, when $x>4.5$, all disturbance modes decrease along streamwise except second order harmonic mode, which makes the hypersonic boundary layer over a blunt wedge under freestream impulse wave being absolutely dominated by second harmonic mode.

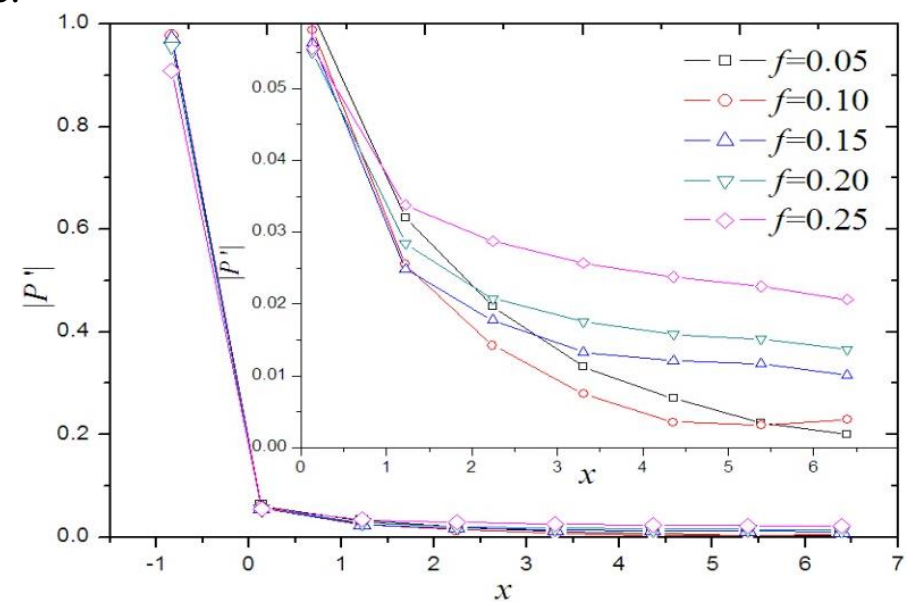

Figure 6. Evolution of pressure disturbances amplitudes for $\mathrm{f} \leq 0.25$ (fundamental frequency and less

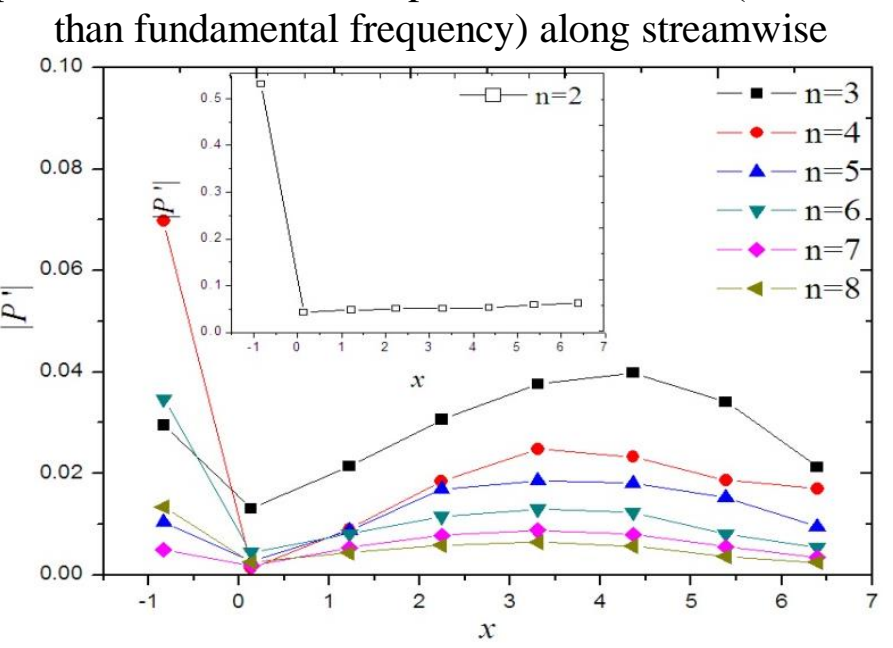

Figure 7. Evolution of pressure disturbances amplitudes for harmonic frequencies (f2-8) along streamwise 


\section{Conclusion}

To investigate the effect of freestream impulse wave on hypersonic flow, the direct numerical simulation is introduced to simulate the response of hypersonic blunt wedge to impulse wave. The interaction between the impulse wave and hypersonic flowfield is analyzed. The evolution of disturbance wave modes in boundary layers is investigated. The conclusions show that:

1) Under freestream impulse wave, the thermodynamic mechanism in boundary layer and the thermal state on wall surface is sharply changed. The interactions between impulse wave and bow shock are very strong. The bow shock is bent obviously outwards when being subjected to freestream impulse wave. The variation of shock location is similar to a damped motion during the interaction process. The shear flow structure in boundary layer is affected by freestream wave, and the effect of the reflection wave between bow shock and wall on shear flow structure is larger than that of impulse wave.

2) After the freestream wave interacting with bow shock and then entering the boundary layer, both the disturbance waves with frequency less than fundamental mode $(f<0.25)$ and harmonic frequency modes in hypersonic boundary layer is induced. In the nose boundary layer, all modes (both fundamental mode and harmonic modes) decrease rapidly along streamwise. When $0<x<4$, all harmonic modes have a rise, however, fundamental mode $\left(f_{1}=0.25\right)$ and the disturbance waves with frequency less than fundamental frequency $(f<0.25)$ in boundary layer along streamwise still keep decreasing although the decay rates becoming small. We prefer to maintain that the rise of harmonic modes and the decay rates of low frequency $(f \leq 2.5)$ becoming small caused by flow recompression, or at least the recompression plays a significant role in the evolution of disturbance waves in boundary layer. In nose region, low frequency disturbances (fundamental mode and the disturbance waves with frequency less than fundamental mode) are the dominant modes in boundary layer; whereas, when $x>2.3$, the dominant mode in boundary layer is transferred from the fundamental mode to the second order harmonic mode. When $x>4.5$, all disturbance modes decrease along streamwise except the second order harmonic mode.

\section{Acknowledgments}

This paper is funded by Beijing Spacecrafts. The authors acknowledge China Academy of Space Technology.

\section{References}

[1] B. Venukumar, G. Jagadeesh, K. P. J. Reddy, Counter flow drag reduction by supersonic jet for a blunt body in hypersonic flow, Physics of Fluids, 18,11 (2006):571-576.

[2] B. P. Harold, James C, Manning Experimental investigation of blast loading on an airfoil in Mach number 0.7 airflow with initial angle of attack change 20, NASA T N-D 1603 (1963).

[3] X. Zhong, High-Order Finite-Difference Schemes for Numerical Simulation of Hypersonic Boundary-Layer Transition, Journal of Computational Physics. 144, 2(1998):662-709.

[4] You Yang, Buanga Björn, Hannemann Volker, etal. Evaluation of Turbulence Models in Predicting Hypersonic and Subsonic Base Flows Using Grid Adaptation Techniques, Chinese Journal of Aeronautics. 25( 2012):325-334.

[5] Y. B. Ma, X. L. Zhong, Boundary-layer receptivity of Mach 7.99 flow over a blunt cone to free-stream acoustic waves, J Fluid Mech. 556(2006):55-103.

[6] V. B. Andrey, Receptivity of a flat plate boundary layer to a free stream axial vortex, European Journal of Mechanics B/Fluids. 21(2002):325-340.

[7] X. Zhong, Leading-Edge Receptivity to Free Stream Disturbance Waves for Hypersonic Flow over a Parabola, Journal of Fluid Mechanics. 441(2001):315-367. 
[8] K.J.A.Westin, A.A. Bakchinov, V.V. Kozlov, P.H. Alfredsson, Experiments on localised disturbances in a flat plate boundary layer. Part 1: The receptivity and evolution of a localised free stream disturbance, Eur. J. Mech. B/Fluids. 17, 6 (1998):823-846.

[9] Y. Ma, X. Zhong, Receptivity of a Supersonic Boundary Layer over a Flat plate. Part 2: Receptivity to Freestream Sound, Journal of Fluid Mechanics. 488(2003): 79-121.

[10] A.V. Fedorov, A. P. Khokhlov, Receptivity of Hypersonic Boundary Layer to Wall Disturbances, Theoretical and Computational Fluid Dynamics. 15(2002):231-254.

[11] A. Tumin, X. Wang, X. Zhong, Numerical Simulation and Theoretical Analysis of Perturbations in Hypersonic Boundary Layers, AIAA Journal. 49, 3, (2011):463-471.

[12] X. Wang, X. Zhong, Y. Ma, Response of a Hypersonic Boundary Layer toWall Blowing-Suction, AIAA Journal. 49, 7(2011):1336-1353.

[13] I. V. Egorov, A. V. Fedorov, V. G. Sudakov, Direct Numerical Simulation of Disturbances Generated by Periodic Suction-Blowing in a Hypersonic Boundary Layer, Theoretical and Computational Fluid Dynamics. 20,1,( 2006):41-54.

[14] J. Steger, R. F. Warming, Flux vector splitting of the in-viscid gasdynamic equations with application to finite difference methods, Journal of Computational Physics. 40(1981):263-293.

[15] X. D. Liu, S. Osher, T. Chan, Weighted essentially non-oscillatory schemes, Journal of Computational Physics. 115(1994):200-212.

[16] Y. Zhang, D. Fu, Y. Ma, et al, Receptivity to free-stream disturbance waves for hypersonic flow over a blunt cone, Science in China Series G: Physics, Mechanics \& Astronomy. 51, 11, (2008):1682-1690.

[17] Shu, Chi-Wang, Essentially Non-Oscillatory and Weighted Essentially Non-Oscillatory Schemes for Hyperbolic Conservation Laws. NASA/CR-97-206253.

[18] X. Tang, H. Lv, X. Meng, et al. Stability characteristic of hypersonic flow over a blunt wedge under freestream pulse wave, Central European Journal of Physics. 12, 1(2014):17-31.

[19] K. F. Stetson, E. R. Thompson, J. C. Donaldson, et al, Laminar boundary layer stability experiments on a cone at Mach 8.Part 2: Blunt cone. AIAA Paper, (1984): 84-0006.

[20] A. Prakash, N. Parsons, X. Wang, X. Zhong, High-order shock-fitting methods for direct numerical simulation of hypersonic flow with chemical and thermal nonequilibrium, Journal of Computational Physics. 230 (2011): 8474-8507.

[21] Y. Zhang, D. Fu, Y. Ma, et al. Receptivity to free-stream disturbance waves for hypersonic flow over a blunt cone, Science in China Series G: Physics, Mechanics \& Astronomy. 51, 11, (2008):1682-1690.

[22] A. J. Laderman, Effect of wall temperature on a supersonic turbulent boundary layer, AIAA Journal. 16, 7(1978): 723-729.

[23] Serkan Özgen, Effect of heat transfer on stability and transitioncharacteristics of boundary-layers, International Journal of Heat and Mass Transfer. 47(2004):4697-4712.

[24] E. M. Hirschel, Basics of Aerothermodynamics. (Springer, Berlin, 2010)

[25] L. Xian, X. L Li, D. X. Fu, Y. W. Ma, Effects of wall temperature on boundary layer stability over a blunt cone at Mach 7.99, Computers \& Fluids. 39(2010):359-371. 